\title{
Das Romseminar für Studierende der Mathematik und Informatik
}

\author{
Markus Haase, Michael Korey, Rainer Nagel, Gregor Nickel und Markus Wacker
}

Das vom Tübinger Funktionalanalytiker Rainer Nagel vor einem Vierteljahrhundert initiierte „Romseminar“ für Studierende der Mathematik und Informatik wird seit nunmehr 14 Jahren in Kooperation an den Universitäten Kiel, Siegen und Tübingen sowie an der HTW Dresden und zusammen mit den Staatlichen Kunstsammlungen Dresden veranstaltet. Das Seminar bietet den Teilnehmenden die besondere Möglichkeit, über den Tellerrand des eigenen Studienfachs hinauszuschauen und sich mit Studierenden anderer Hochschulen auszutauschen. Daneben geht es darum, Präsentation, Rhetorik und Diskussion in einem fachlichen Kontext zu üben. Im Laufe des Wintersemesters wird zunächst das jährlich wechselnde Seminarthema durch gemeinsame Lektüre, Diskussionen und gelegentliche Gastvorträge erschlossen. Bis Ende Dezember werden Themen für die Präsentationen individuell definiert und schließlich im Dialog mit der Gruppe vor Ort erprobt. Die räumlich verteilte Vorarbeit mündet in eine einwöchige, gemeinsame Exkursion nach Rom, wo die Teilnehmenden an der Accademia Nazionale dei Lincei ihre Gedanken präsentieren und intensiv miteinander diskutieren. Dabei lassen wir uns durch ein vielfältiges Begleitprogramm auch an sonst touristisch nicht zugänglichen Orten dieser „Ewigen Stadt" inspirieren. Voraussetzung für das Seminar ist die Bereitschaft, sich mit der Thematik engagiert auseinanderzusetzen sowie die eigene kritische Urteilsfähigkeit zu schärfen. Ein Eindruck von den bisherigen Themen und dem pädagogischen Konzept kann durch einen Besuch der Webseite www.math.uni-tuebingen.de/de/forschung/agfa/ romseminare oder durch den Bericht von Karl H. Hofmann in den Mitteilungen 13-3 (2005) gewonnen werden.

Das Thema des Romseminars 2020 war „Digitalisierung - Wie diskret wollen wir leben? Annäherungen durch Mathematik und Informatik", eine Thematik, die eine noch am Anfang des WS 2019/20 ungeahnte Brisanz auch im Bereich der (akademischen) Lehre bekommen würde. Das Seminar hat sich dem Schlagwort „Digitalisierung“ auf zwei Ebenen gewidmet. Zum einen wurden zentrale Begriffe bzw. Phänomene im Kontext der Digitalisierung geklärt. Dies betraf u.a. Themen wie „Big Data“, „Künstliche Intelligenz" oder „Autonome Maschinen“. Hier ging es also darum, die technische Seite der Digitalisierung möglichst so transparent zu machen, dass deren begriffliche Voraussetzungen und technische Möglichkeiten beurteilt werden können, ohne auf tendenziöse (teils dystopisch-dramatisierende, teils euphorisch-werbende) Interpretationen angewiesen zu sein. Zum anderen ging es um einen vertiefenden Blick auf ausgewählte gesellschaftliche Bereiche, die durch den Prozess der Digitalisierung wesentlich umgestaltet werden. Das Themenspektrum war dementsprechend facettenreich, es umfasste einerseits studentische Vorträge $\mathrm{zu}$ den Grundlagen von neuronalen Netzen, der ${ }_{5} \mathrm{G}$-Technik, physikalischen Voraussetzungen der Mikroelektronik oder zu dem Hintergrund von „Kryptowährungen“. Andererseits wurden gesellschaftliche Dimensionen diskutiert: von social bots und social scoring über Digitalisierung in der Arbeitswelt, im Bildungssystem und im Sport bis hin zur digitalen Umgestaltung von Mobilität und Kriegführung.

Einer der Teilnehmer, der Tübinger Student Patrick Hermle, wählte für sein Referat die Form eines szenischen Monologs, in dem er reflektiert, was der Vormarsch des Digitalen für das Selbstverständnis eines Mathematikers bedeuten könnte. Seine Reflexionen über das eigene Tun haben sowohl das studentische Publikum als auch uns Organisatoren beeindruckt, deshalb haben wir nach dem Seminar die Herausgeber der Mitteilungen auf seinen Text aufmerksam gemacht.

Prof.Dr. Markus Haase, haase@math.uni-kiel.de

Dr. Michael Korey, michael.korey@skd.museum

Prof. Dr. Rainer Nagel, rana@fa.uni-tuebingen.de

Prof.Dr. Gregor Nickel, nickel@mathematik.uni-siegen.de

Prof.Dr.Markus Wacker,wacker@informatik.htw-dresden.de 